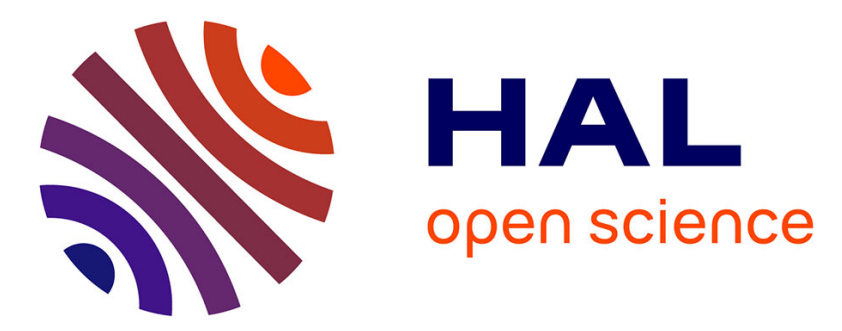

\title{
Tools for improving feeding strategies in a SBR with several species
}

\author{
Pedro Gajardo, Hector Ramirez Cabrera, Julio C. Rodriguez
}

\section{To cite this version:}

Pedro Gajardo, Hector Ramirez Cabrera, Julio C. Rodriguez. Tools for improving feeding strategies in a SBR with several species. Bioprocess and Biosystems Engineering, 2014, 37 (1), pp.63 - 70. 10.1007/s00449-013-1077-1 . hal-01089424

\section{HAL Id: hal-01089424 \\ https://inria.hal.science/hal-01089424}

Submitted on 11 Dec 2014

HAL is a multi-disciplinary open access archive for the deposit and dissemination of scientific research documents, whether they are published or not. The documents may come from teaching and research institutions in France or abroad, or from public or private research centers.
L'archive ouverte pluridisciplinaire HAL, est destinée au dépôt et à la diffusion de documents scientifiques de niveau recherche, publiés ou non, émanant des établissements d'enseignement et de recherche français ou étrangers, des laboratoires publics ou privés. 


\begin{abstract}
Received: date / Accepted: date
Abstract This paper analyzes feeding strategies in a sequential batch reactor (SBR) with the objective of reaching a given (low) substrate level as quickly as possible for a given volume of water. Inside the SBR, several species compete for a single substrate, which leads to a minimal time control problem in which the control variable is the feeding rate. Following [10], we allow the control variable to be a bounded measurable function of time combined with possible impulses associated with instantaneous dilutions. For this problem, the extremal trajectories of the singular arc type are characterized as the strategies used to maintain the substrate at a constant level. Because this optimization problem is difficult to solve, this characterization provides a valuable tool for investigating the optimality of various feeding strategies. Our aim is thus to illustrate the use of this tool by proposing potential optimal feeding strategies, which may then be compared with other more intuitive strategies. This aim was accomplished via several numerical experiments in which two specific strategies are compared.
\end{abstract}

Supported by FONDECYT under grant No. 1120239 (P. Gajardo), 1110888 (H. Ramírez) and 1130538 (J.C. Rodríguez), the BASAL Project (Centro de Modelamiento Matemático, Universidad de Chile), CONICYT Anillo ACT1106, project BIONATURE of CIRIC, INRIA-Chile, and UFAM-INCTMat Avanço Global e Integrado da Matemática Brasilera e Contribuções a Região, CAPES-Brasil.

Pedro Gajardo

Departamento de Matemática, Universidad Técnica Federico Santa María, Avda. España 1680, Valparaíso - Chile

Tel.: +56-32-2654130

E-mail: pedro.gajardo@usm.cl

Héctor Ramírez C.

Departamento de Ingeniería Matemática, Universidad de Chile \& Centro de Modelamiento Matemático, Universidad de Chile-CNRS, Casilla 170/3, Santiago - Chile Tel.: +56-2-29784980

E-mail: hramirez@dim.uchile.cl

Julio C. Rodríguez

Departamento de Matemáticas, Universidad de Valparaíso, Casilla 5030, Valparaíso - Chile Tel.: +56-32-2508274

E-mail: julio.rodriguez@uv.cl 
Keywords Sequential batch reactors - Minimal time problem - Singular arc strategies

\section{Introduction}

Sequential batch reactors (SBRs) typically consist of a tank filled with biological micro-organisms that are capable of degrading an undesirable substrate. These devices are often used in biotechnological applications, most notably in wastewater treatments (see $[10,14,18,22]$ for details about the fundamental role of SBRs in bioengineering). For this purpose, we use the typical sequence of cycles, which is composed of three phases:

- Phase 1: fill the reactor with water to be treated,

- Phase 2: wait for the concentration of the undesirable substrate to decrease to a given (low) concentration,

- Phase 3: remove the clean water from the reactor, leaving the sludge inside.

In this paper, we focus on a SBR in which several species compete for a single substrate and on the corresponding optimal control problem of feeding to minimize the time in which the SBR reaches a desired (low) concentration of the substrate (i.e., Phases 1 and 2 described above). This optimal control problem is the same as that presented in $[10,11]$, which corresponds to an extension of the one-species model introduced in [18]. This problem has been solved for certain cases; for instance, explicit optimal feeding strategies are given in [18], in which the SBR contains only one species. Feeding strategies are also given in [10], in which the SBR contains two species with nondecreasing growth functions. See also $[2,20]$ for other related feeding problems involving SBRs. Nonetheless, bioprocesses generally involve a significant amount of biodiversity, particularly in wastewater treatment. Microbial communities can contain thousands of species [9]. Thus, it is relevant to study optimal feeding strategies in the presence of several species.

This multi-species setting leads to an optimization problem that is difficult to solve. However, [11] presented a method for characterization when a singular arc is presented in an optimal feeding strategy. Indeed, this characterization establishes that an extremal curve (eventually optimal) is an singular arc if and only if the substrate level remains constant during that period of time. Because the existence of singular arcs can lead to complex solutions that are not easily tractable from a mathematical point of view, this characterization is very valuable for verifying the optimality of various feeding strategies. We thus provide explicit conditions for which the above-mentioned characterization holds. These conditions depend on the growth functions of the species involved in the depollution process. When the appropriate conditions are fulfilled, we can propose potential optimal feeding strategies, based on the existence of a singular arc, and can compare them with other more intuitive strategies via numerical simulations. 


\section{Mathematical model and Pontryagin's principle}

In this section, we define the optimal control problem studied in this article. This problem was introduced by Gajardo, Ramírez and Rapaport [10], as an extension of the minimal time problem presented by Moreno in [18]. We finish this section by exhibiting some basic results concerning Pontryagin's principle, which permits us, in the next section, to understand the meaning of a singular arc strategy in this problem.

The dynamics of a SBR with several species can be described as follows (see [22]):

$$
\left\{\begin{array}{l}
\dot{x}_{i}=\mu_{i}(s) x_{i}-\frac{F}{v} x_{i}, x_{i}\left(t_{0}\right)=x_{i 0} \quad(i=1 \cdots n), \\
\dot{s}=-\sum_{j=1}^{n} \mu_{j}(s) x_{j}+\frac{F}{v}\left(s_{i n}-s\right), s\left(t_{0}\right)=s_{0} \\
\dot{v}=F, v\left(t_{0}\right)=v_{0},
\end{array}\right.
$$

where $x_{i}, s$ and $v$ represent the concentration of the $i$ th species, the concentration of the substrate and the current volume of water in the tank, respectively. The parameter $s_{i n}>0$ is a constant that represents the substrate concentration in the input flow. The growth functions $\mu_{i}(\cdot)$ are non-negative smooth functions such that $\mu_{i}(0)=0$, and the input flow $F$ is a non-negative control variable.

The aim is to treat the maximum quantity of water in the SBR as quickly as possible. From a mathematical point of view, this means that, given a (desirable) substrate concentration $\left.s_{\text {out }} \in\right] 0, s_{\text {in }}$ [ and the volume of the reactor $v_{\max }$, we want to solve the following minimal time problem:

$$
\inf _{F(\cdot)}\left\{t-t_{0} \mid s^{t_{0}, \xi, F}(t) \leq s_{\text {out }}, v^{t_{0}, \xi, F}(t)=v_{\max }\right\},
$$

where $s^{t_{0}, \xi, F}(\cdot), v^{t_{0}, \xi, F}(\cdot)$ denote solutions of (2.1) with the initial condition $\xi=\left(x_{10}, \ldots, x_{n 0}, s_{0}, v_{0}\right)$ at time $t_{0}$ and when a particular control strategy $F(\cdot)$ is used. It is worth emphasizing that our theoretical results are valid provided that the initial condition $\xi$ is in the domain $\left.\left.\left.\mathcal{D}=\left(\mathbb{R}_{+}^{n} \backslash\{0\}\right) \times\right] 0, s_{i n}\right] \times\right] 0, v_{\text {max }}[$.

Here, $F(\cdot)$ is allowed to be a non-negative measurable function combined with possible positive impulses; that is, $d F(t)=u(t) d t+d \sigma$, where $u(\cdot)$ is a measurable non-negative control that we impose to be bounded from above by some positive value $u_{\max }$ because it corresponds to the use of a pump device, and at time $t$, the non-negative impulse $d \sigma$ corresponds to an instantaneous addition of volume. The latter is useful for modeling practical situations in which large amounts of polluted water are added to the SBR in a short time (e.g., $[5,7])$.

From [10], we know that a time parameterization $\tau \geq t_{0}$ such that $d t=$ $r(\tau) d \tau$ with

$$
r(\tau)=\left\{\begin{array}{l}
1, \text { when the pump device is used } \\
0, \text { when an impulse is used }
\end{array}\right.
$$


allows us to replace the dynamics (2.1) by the system

$$
\left\{\begin{array}{l}
\frac{d x_{i}}{d \tau}=r \mu_{i}(s) x_{i}-\frac{u}{v} x_{i} \quad(i=1 \cdots n), \\
\frac{d s}{d \tau}=-r \sum_{j=1}^{n} \mu_{j}(s) x_{j}+\frac{u}{v}\left(s_{i n}-s\right), \\
\frac{d v}{d \tau}=u
\end{array}\right.
$$

where the controls $u(\cdot)$ and $r(\cdot)$ are sought among measurable functions with respect to $\tau$, taking values in $\left[0, u_{\max }\right]$ and $\{0,1\}$, respectively. In this formulation, $u(\cdot)$ acts as an ordinary control when $r=1$ and controls the amplitude of the impulse when $r=0$, with the same single constraint $u \in\left[0, u_{\max }\right]$. Consequently, control $r(\cdot)$ indicates the presence of an instantaneous dilution. This conclusion can also be interpreted from system (2.3) by noting that $r=0$ causes all of the terms related to biological interactions to vanish. Indeed, from a process point of view, one considers impulses when the time needed to add a given volume of water is sufficiently small, such that growth can be neglected during this time. This assumption also allows us to neglect this time in the total time of the process. Thus, we obtain the following value function for the reformulated dynamics $(2.3)$

$$
\begin{aligned}
V(\xi)= & \inf _{(u, r)(\cdot)} \int_{t_{0}}^{\tau} r(\theta) d \theta \text { such that } \\
& s^{t_{0}, \xi, u, r}(\tau) \leq s_{\text {out }}, v^{t_{0}, \xi, u, r}(\tau)=v_{\text {max }},
\end{aligned}
$$

where $s^{t_{0}, \xi, u, r}(\cdot), v^{t_{0}, \xi, u, r}(\cdot)$ denote solutions of (2.3) with the initial condition $\xi \in \mathcal{D}$ at time $t_{0}$ and with controls $u(\cdot)$ and $r(\cdot)$.

Remark 1 As one can always take $r=0$ and $u=0$ for an arbitrarily large $\tau$-interval without modifying the total time, the minimal time problem has no unique solution. Hence, we will be only interested in controls satisfying $r(\tau) \neq 0$ or $u(\tau) \neq 0$ for all time $\tau$.

Now, set $\mathbf{z}=\left(x_{1}, \cdots, x_{n}, s, v\right)^{\top}, u_{1}=r$ and $u_{2}=u$.

In [11], the Pontryaguin principle (cf. [19]) applied to problem (2.4) can be stated as follows:

If $\boldsymbol{u}_{*}=\left(u_{1 *}, u_{2 *}\right)$ is an optimal control and $\mathbf{z}_{*}$ is its corresponding trajectory on $\mathbb{R}^{n+2}$, then there exists a constant $\lambda_{0} \geq 0$ and an absolutely continuous function $\tau \mapsto \boldsymbol{\lambda}(\tau)=\left(\lambda_{1}(\tau), \ldots, \lambda_{n+2}(\tau)\right)$ such that for almost every $\tau,\left(\boldsymbol{\lambda}, \lambda_{0}\right)$ never vanish and satisfy

$$
\dot{\mathbf{z}}=\frac{\partial \mathcal{H}}{\partial \boldsymbol{\lambda}}\left(\mathbf{z}, \boldsymbol{\lambda}, \boldsymbol{u}_{*}\right), \quad \dot{\boldsymbol{\lambda}}=-\frac{\partial \mathcal{H}}{\partial \boldsymbol{z}}\left(\mathbf{z}, \boldsymbol{\lambda}, \boldsymbol{u}_{*}\right),
$$

where the Hamiltonian $\mathcal{H}$ is given by

$$
\mathcal{H}\left(\boldsymbol{z}, \boldsymbol{\lambda}, u_{1}, u_{2}\right)=u_{1} \phi_{u_{1}}(\mathbf{z}, \boldsymbol{\lambda})+u_{2} \phi_{u_{2}}(\mathbf{z}, \boldsymbol{\lambda}) .
$$


Here, we define the following functions

$$
\left\{\begin{aligned}
\phi_{u_{1}}(\mathbf{z}, \boldsymbol{\lambda}) & :=\lambda_{0}+\sum_{i=1}^{n} \mu_{i}(s) x_{i}\left(\lambda_{i}-\lambda_{n+1}\right), \\
\phi_{u_{2}}(\mathbf{z}, \boldsymbol{\lambda}) & :=-\sum_{i=1}^{n} \frac{x_{i}}{v} \lambda_{i}+\frac{\left(s_{i n}-s\right)}{v} \lambda_{n+1}+\lambda_{n+2} .
\end{aligned}\right.
$$

Furthermore, the optimal control $\boldsymbol{u}_{*}$ minimizes the Hamiltonian over the control admissible set, through the curve $(\boldsymbol{\lambda}(\tau), \mathbf{z}(\tau))$.

For the sake of simplicity, we will simply use $\phi_{u_{i}}(\tau)$ instead of $\phi_{u_{i}}(\mathbf{z}(\tau), \boldsymbol{\lambda}(\tau))$, and we assume that $\lambda_{0}=1$. The latter condition corresponds to the normal extremals.

\section{Characterization of singular arcs}

In our context, a singular arc corresponds to an extremal curve for which there exists a nontrivial interval $\left[\tau_{1}, \tau_{2}\right] \subset[0, T]$ where both switching functions $\phi_{u_{i}}(\tau), i=1,2$, are identically zero. This can be understood as an extension of the standard definition of singular arcs when only one control is considered (e.g., [3, Part III, Ch. 2]).

When a singular arc is presented in the optimal strategy, minimization of the Hamiltonian $\mathcal{H}$ introduced in (2.6) can lead to complex feeding strategies. The next result thus provides important qualitative information about singular arcs, constraining them only to extremal curves for which the substrate level $s$ remains constant. This constraint permits us to limit our study of possible optimal feeding strategies.

Singular strategies have been known to appear in many control problems of particular interest to chemical engineers and have already been addressed with the help of optimal control theory $[2,6,13,14,20]$. In addition, [8] and [21] have demonstrated the possible appearance of singular arcs in the start-up of bioreactors and in a variety of chemical reactor optimization problems.

The issue of singular arcs is well-known in the classical theory of optimal control; see, for instance, [15]. Currently, this type of trajectory plays an important role in optimal control theory. These trajectories are studied in high-order optimality conditions; see, for instance, [4], [12,16].

The next characterization of singular arcs was reported in [11].

Theorem 1 Suppose that the matrix

$$
\mathfrak{D}(s)=\left(\begin{array}{ccc}
\mu_{1}^{(1)}(s) & \cdots & \mu_{n}^{(1)}(s) \\
\mu_{1}^{(2)}(s) & \cdots & \mu_{n}^{(2)}(s) \\
\vdots & \ddots & \vdots \\
\mu_{1}^{(n)}(s) & \cdots & \mu_{n}^{(n)}(s)
\end{array}\right)
$$


is nonsingular for any $s \in\left(0, s_{i n}\right)$. Here, $\mu_{i}^{(j)}$ denotes the $j$-th derivative of function $\mu_{i}$. Then, an extremal curve is a singular arc on $\left(\tau_{1}, \tau_{2}\right) \subset[0, T]$ if and only if $s(\cdot)$ is constant on $\left(\tau_{1}, \tau_{2}\right)$.

Remark 2 The characterization stated above ensures that any optimal strategy is composed of bang-bang controls (which, in our case, means either to close the pump or to do one impulsion) or that the pump is used in order to maintain a constant substrate level, where the latter approach corresponds to a singular arc. However, this characterization does not prevent the presentation of more than one singular arc. For the sake of simplicity, in the next sections, we only consider optimal strategies containing at most one singular arc.

\section{Applications}

In this section, we apply Theorem 1 to study singular arcs for species with particular biological characteristics that have not been considered in previous articles $[10,18]$. This tool allows us to propose potential optimal feeding strategies, which can then be compared with other more intuitive strategies.

4.1 Arbitrary number of species following Monod growth functions

Suppose that each of the $n$ species of microorganisms in competition for a single substrate in the SBR follows a Monod law growth function [17], that is

$$
\mu_{i}(s)=\frac{\mu_{\max , i} s}{K_{i}+s}, \quad i=1, \cdots, n
$$

where, for the $i$-species, $\mu_{\max , i}$ and $K_{i}$ represent the maximal growth rate and the half saturation constant or Monod constant, respectively, and $K_{i} \neq K_{j}$, for all $i \neq j$. In this case, it is easy to verify that the hypothesis of Theorem 1 holds true (cf. Proposition 3.5 of [11]), and we obtain the following result:

Proposition 2 Under the hypotheses of this subsection, an extremal curve is a singular arc on $\left(\tau_{1}, \tau_{2}\right) \subset[0, T]$ if and only if $s(\cdot)$ is constant on $\left(\tau_{1}, \tau_{2}\right)$.

4.2 Two species with a Haldane and a Monod growth function

Consider now that one of the two species has a Monod growth law:

$$
\mu_{M}(s)=\frac{\mu_{\max , M} s}{K_{M}+s},
$$

(where, as before, $\mu_{\max , M}$ is the maximal growth rate and $K_{M}$ is the half saturation constant or Monod constant) and the other species follows a Haldane growth law [1], which is usually used to represent a substrate inhibiting growth at high values, as given by

$$
\mu_{H}(s)=\frac{\mu_{\max , H} s}{K_{S}+s+\frac{s^{2}}{K_{I}}}
$$


where $\mu_{\max , H}$ is the maximal growth rate, $K_{I}$ is an inhibition constant and $K_{S}$ is the affinity or saturation constant.

In this situation, we can verify the following result:

Proposition 3 The hypothesis of Theorem 1 is fulfilled if one of the following conditions is satisfied.

(1) $K_{I}=K_{M}$ and $4 K_{S}<K_{I}$.

(2) $K_{I}>K_{M}$ and $K_{I} K_{S}=K_{M}\left(K_{I}-K_{M}\right)$.

(3) $K_{I}-K_{M}=2 K_{S}$ and $K_{I} K_{S}<K_{M}\left(K_{I}-K_{M}\right)$.

(4) $\frac{K_{I}-K_{M}}{K_{S}}>\max \left\{2, \frac{K_{I}}{K_{M}}\right\}$.

Conditions (1)-(4) of Proposition 3 are derived from an algebraic analysis of the determinant of the matrix $\mathfrak{D}(s)$ given in (3.1). Consequently, we obtain

Corollary 4 Under any of the conditions of Proposition 3, an extremal curve is a singular arc on $\left(\tau_{1}, \tau_{2}\right) \subset[0, T]$ if and only if $s(\cdot)$ is constant on $\left(\tau_{1}, \tau_{2}\right)$.

4.3 Two species with a linear and a Monod growth function

Consider again two species, one following a Monod growth law, cf. (4.2), and one following a linear law given by

$$
\mu_{L}(s)=c s
$$

where $c$ is a positive constant. This type of function can model very large growth functions (for instance, either Monod or Haldane type) at the beginning of its range.

In this situation, the determinant of $\mathfrak{D}(s)$, defined in (3.1), is given by

$$
\frac{2 c \mu_{\max , M}}{\left(K_{M}+s\right)^{3}}
$$

Therefore, the hypothesis of Theorem 1 is always satisfied.

Proposition 5 Under the hypotheses of this subsection, an extremal curve is a singular arc on $\left(\tau_{1}, \tau_{2}\right) \subset[0, T]$ if and only if $s(\cdot)$ is constant on $\left(\tau_{1}, \tau_{2}\right)$.

4.4 Two species with a linear and a Haldane growth function

Finally, we consider two species, one following a linear growth law, cf. (4.4) and one following a Haldane growth law, cf. (4.3). Hence, we can verify that the hypothesis of Theorem 1 is fulfilled, provided that $\mu_{H}^{\prime \prime}(s) \neq 0$, for all $s \in\left(0, s_{i n}\right)$. Indeed, the determinant of the matrix $\mathfrak{D}(s)$ is exactly $c \mu_{H}^{\prime \prime}(s)$. Consequently, we find 
Pedro Gajardo et al.

Corollary 6 For the two-species case considered in this subsection, suppose that $\mu_{H}^{\prime \prime}(s) \neq 0$, for all $s \in\left(0, s_{i n}\right)$. Then, an extremal curve is a singular arc on $\left(\tau_{1}, \tau_{2}\right) \subset[0, T]$ if and only if $s(\cdot)$ is constant on $\left(\tau_{1}, \tau_{2}\right)$.

\section{Numerical simulations}

In this section, we compare two feeding strategies for the cases stated in Section 4; namely, we compare the immediate one-impulse strategy and the singular arc strategy. As discussed in previous articles $[10,11,18]$, these two types of strategies naturally arise as feeding strategies in different bioprocesses.

The immediate one-impulse strategy (denoted IOI for short) was introduced in [10], and it consists of introducing, from a given initial state $\xi=$ $\left(x_{10}, \ldots, x_{n 0}, s_{0}, v_{0}\right)$ at time $t_{0}$, the following: 1$)$ An impulse of volume $v_{\max }-$ $v_{0}$ at $t_{0}$. This can be achieved by $r(\tau)=0, u(\tau)=u_{\max }$, for $\tau \in\left[t_{0}, t_{0}+\right.$ $\left.\left(v_{\max }-v_{0}\right) / u_{\max }\right]$, and 2) A null control (no feeding) until the concentration $s(\tau)$ reaches $s_{\text {out }}$.

The singular arc strategy has been previously analyzed $[10,18]$ and depends on a given value $s^{*}$ for the substrate (indeed, it is denoted by $S A\left(s^{*}\right)$ for short). Roughly speaking, this strategy consists of reaching, as quickly as possible, a given level $s^{*}$ in $\left(s_{\text {out }}, s_{\text {in }}\right)$ and then maintaining $s$ constant and equal to $s^{*}$ until $v$ reaches $v_{\max }$. Finally, we set $u=0$ and $r=1$ (which means the pump is closed) until $s$ reaches $s_{\text {out }}$. In all of our numerical experiments, the value of $s^{*}$ is chosen to minimize the cost of this type of strategy among all possible values of $s^{*}$ in $\left(s_{\text {out }}, s_{\text {in }}\right)$.

Recall that in the case of two species with increasing growth functions, it was shown in [10] that these two strategies were unique possible optimal strategies for our optimal control problem.

As an example, we first consider the following three Monod growth functions (see Figure 1):

$$
\mu_{1}(s)=\frac{s}{1+s}, \mu_{2}(s)=\frac{2 s}{1.5+s}, \mu_{3}(s)=\frac{4 s}{2+s},
$$

as well as the following parametric values $v_{\max }=10, s_{\text {out }}=0.1$ and $s_{\text {in }}=5$ and the initial conditions $x_{10}=1, x_{20}=0.001, s_{0}=3$ and $v_{0}=1$. In Table 1, we compare, for different values of the initial condition $x_{30}$, the times achieved by the IOI and $S A\left(s^{*}\right)$ strategies. Thus, the results reported in Table 1 establish that $S A\left(s^{*}\right)$ exhibits an improvement close to $25 \%$ with respect to IOI for some values of $x_{30}$. We thus conclude that IOI is not optimal for this particular setting.

Note that it is already known (see [10]) that when there are only two Monod growth functions in the bioreactor and when one of them is clearly more performant than the other (i.e., its graphical image is always higher), the IOI strategy is indeed optimal. Thus, the previous results indicate that considering a third species (even a more performant one) can lead to a loss of the optimality of IOI. We believe that this fact can be important in practice. 


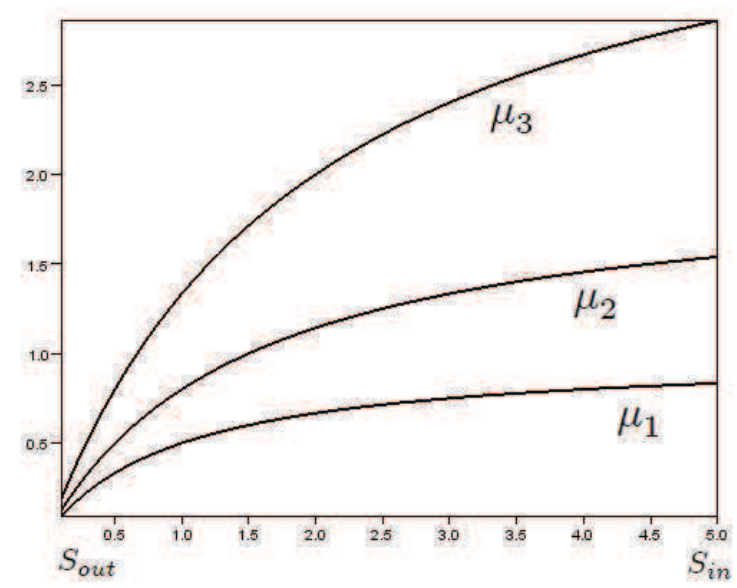

Fig. 1 Graphs of the three growth functions considered in the example.

The case of an arbitrary number of species following increasing growth functions was analyzed in [11]. Therein, conditions that ensure the optimality of the IOI strategy were studied.

Table 1.

\begin{tabular}{|c||c|c|c|c|}
\hline$x_{30}$ & $T(I O I)$ & $s^{*}$ & $T\left(S A\left(s^{*}\right)\right)$ & $\frac{(\mathrm{T}(\mathrm{IOI})-\mathrm{T}(\mathrm{SA}))}{\mathrm{T}(\mathrm{IOI})}$ \\
\hline $10^{-4}$ & 5.416174 & 4.226000 & 5.402767 & $0.2 \%$ \\
$10^{-3}$ & 5.389022 & 3.540000 & 4.978126 & $7.6 \%$ \\
$10^{-2}$ & 5.172141 & 3.442000 & 4.170769 & $19.4 \%$ \\
0.05 & 4.669824 & 3.344000 & 3.548414 & $24.0 \%$ \\
0.1 & 4.350146 & 3.246000 & 3.274169 & $24.7 \%$ \\
0.5 & 3.458854 & 3.050000 & 2.620281 & $24.2 \%$ \\
\hline
\end{tabular}

For the rest of the cases, we consider two species that are in competition for a single substrate in the SBR. In all of these cases, we consider the parametric values $v_{\max }=10, s_{\text {out }}=0.1$ and $s_{\text {in }}=5$ and the initial conditions $x_{10}=1$, $s_{0}=3$, and $v_{0}=1$. We then compare, for different values of $x_{20}$, the times achieved by the $I O I$ and $S A\left(s^{*}\right)$ strategies.

In Table 2, we illustrate the case in which one species follows a Haldane growth law and the other follows a Monod growth law. These growth functions fall into some of the cases of Proposition 3 (see Figure 2). 
Table 2 .

\begin{tabular}{|c|c|c|c|c|}
\hline \multicolumn{5}{|c|}{$K_{I}=K_{M}, 4 K_{S}<K_{I}: \mu_{H}(s)=\frac{2 s}{1+s+\frac{s^{2}}{6}}$, and $\mu_{M}(s)=\frac{4 s}{6+s}$} \\
\hline$x_{20}$ & $T(I O I)$ & $s^{*}$ & $T\left(S A\left(s^{*}\right)\right)$ & $\frac{(\mathrm{T}(\mathrm{IOI})-\mathrm{T}(\mathrm{SA}))}{\mathrm{T}(\mathrm{IOI})}$ \\
\hline $10^{-4}$ & 3.914240 & 2.462000 & 3.716620 & $5.0487 \%$ \\
\hline $10^{-3}$ & 3.909991 & 2.462000 & 3.715882 & $4.9644 \%$ \\
\hline $10^{-2}$ & 3.870175 & 2.462000 & 3.708550 & $4.1762 \%$ \\
\hline 0.05 & 3.73 & 2.658000 & 3.673 & $1.5937 \%$ \\
\hline 0.1 & 3.612956 & 4.716000 & 3.613056 & $-0.0028 \%$ \\
\hline 0.5 & 3.162882 & 4.716000 & 3.163642 & $-0.0240 \%$ \\
\hline \multicolumn{5}{|c|}{$\frac{K_{I}-K_{M}}{K_{S}}>\max \left\{2, \frac{K_{I}}{K_{M}}\right\}: \quad \mu_{H}(s)=\frac{2 s}{\frac{6}{5}+s+\frac{s^{2}}{6}}, \quad \mu_{M}(s)=\frac{4 s}{2+s}$} \\
\hline$x_{20}$ & $T(I O I)$ & $s^{*}$ & $T\left(S A\left(s^{*}\right)\right)$ & $\frac{(\mathrm{T}(\mathrm{IOI})-\mathrm{T}(\mathrm{SA}))}{\mathrm{T}(\mathrm{IOI})}$ \\
\hline $10^{-4}$ & 4.038229 & 2.756000 & 3.912040 & $3.1249 \%$ \\
\hline $10^{-3}$ & 3.832359 & 4.716000 & 3.832356 & $0.0001 \%$ \\
\hline $10^{-2}$ & 3.272825 & 4.716000 & 3.274040 & $-0.0371 \%$ \\
\hline 0.05 & 2.761514 & 4.716000 & 2.762851 & $-0.0484 \%$ \\
\hline 0.1 & 2.529374 & 4.716000 & 2.530603 & $-0.0486 \%$ \\
\hline 0.5 & 1.975955 & 4.716000 & 1.976672 & $-0.0363 \%$ \\
\hline
\end{tabular}
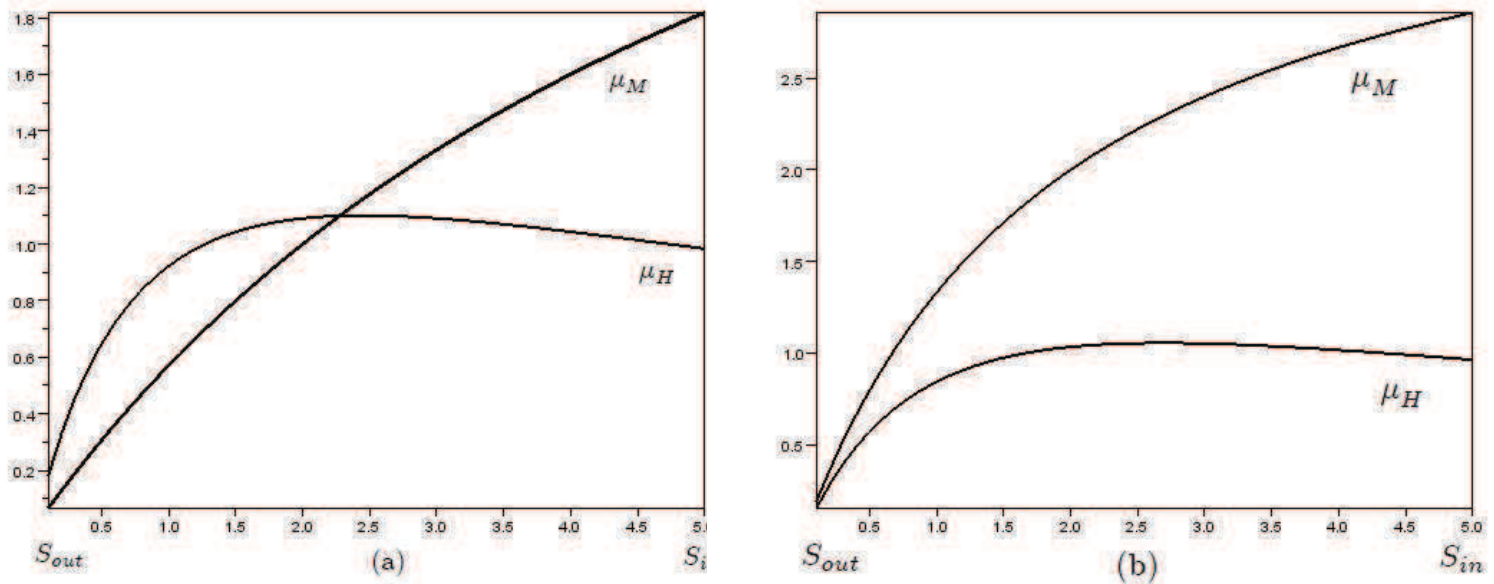

Fig. 2 Two species with Haldane and Monod law growth functions: (a) $K_{I}=K_{M}, 4 K_{S}<$ $K_{I}$, (b) $\frac{K_{I}-K_{M}}{K_{S}}>\max \left\{2, \frac{K_{I}}{K_{M}}\right\}$

In Table 3, we illustrate a situation in which one of the species follows a Monod growth law and the other species follows a linear growth law, given by (see Figure 3-(a))

$$
\mu_{M}(s)=\frac{4 s}{3+s}, \quad \text { and } \quad \mu_{L}(s)=2 s .
$$



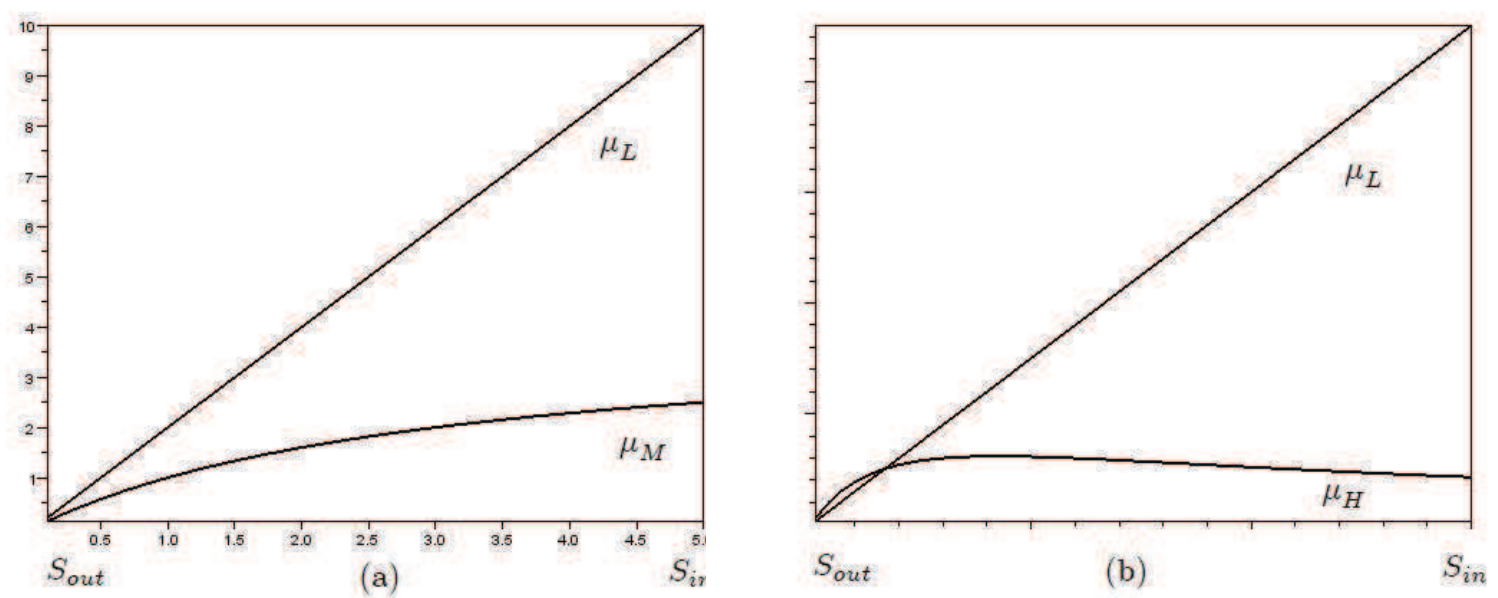

Fig. 3 Two species with growth functions: (a) Linear- Monod (b) Linear-Haldane.

\begin{tabular}{|c||c|c|c|c|}
\hline$x_{20}$ & $T(I O I)$ & $s^{*}$ & $T\left(S A\left(s^{*}\right)\right)$ & $\frac{(\mathrm{T}(\mathrm{IOI})-\mathrm{T}(\mathrm{SA}))}{\mathrm{T}(\mathrm{IOI})}$ \\
\hline $10^{-4}$ & 1.894604 & 4.716000 & 1.897233 & $-0.1388 \%$ \\
$10^{-3}$ & 1.600381 & 4.716000 & 1.603105 & $-0.1702 \%$ \\
$10^{-2}$ & 1.317108 & 4.716000 & 1.319561 & $-0.1862 \%$ \\
0.05 & 1.130736 & 4.716000 & 1.132728 & $-0.1762 \%$ \\
0.1 & 1.052256 & 4.716000 & 1.053935 & $-0.1596 \%$ \\
0.5 & 0.870140 & 4.716000 & 0.870980 & $-0.0965 \%$ \\
\hline
\end{tabular}

In Table 4, we illustrate a situation in which one of the species follows a Haldane growth law and the other species follows a linear growth law, given by (see Figure 3-(b))

$$
\mu_{H}(s)=\frac{6 s}{2+s+\frac{s^{2}}{9}}, \quad \text { and } \quad \mu_{L}(s)=1.5 s
$$

Table 4.
\begin{tabular}{|c|c|c|c|c|}
\hline$x_{20}$ & $T(I O I)$ & $s^{*}$ & $T\left(S A\left(s^{*}\right)\right)$ & $\frac{(\mathrm{T}(\mathrm{IOI})-\mathrm{T}(\mathrm{SA}))}{\mathrm{T}(\mathrm{IOI})}$ \\
\hline $10^{-4}$ & 1.435443 & 4.226000 & 1.434500 & $0.0657 \%$ \\
$10^{-3}$ & 1.429464 & 4.618000 & 1.429450 & $0.0010 \%$ \\
$10^{-2}$ & 1.384587 & 4.716000 & 1.385042 & $-0.0329 \%$ \\
0.05 & 1.289891 & 4.716000 & 1.290788 & $-0.0695 \%$ \\
0.1 & 1.231523 & 4.716000 & 1.232486 & $-0.0782 \%$ \\
0.5 & 1.064653 & 4.716000 & 1.065375 & $-0.0678 \%$ \\
\hline
\end{tabular}

\section{Conclusions}

In this paper, we have studied the optimal control problem consisting of feeding in a SBR in which several species compete for a single substrate, with the 
objective being to reach a given (low) substrate level in a minimal time. Our mathematical model allows for instantaneous dilution of the polluted water as well as continuous feeding from a pump.

For this multi-species setting, the existence of singular arcs is known to be fully characterized as the intervals of time in which the substrate concentration remains constant. This characterization permits us to conclude that optimal feeding strategies are composed of only three types of feeding phases: dilutions, manipulations of the pump to maintain a constant substrate concentration and closing of the pump (which involves waiting for the concentration of the substrate to decrease to a given level). Of course, these three phases can be combined in an infinite number of ways. Our numerical simulations were conducted with the aim of comparing only two feeding strategies: the IOI strategy, in which the tank is filled with a single dilution followed by waiting, and the singular arc strategy, in which, a given substrate concentration is reached as quickly as posible and the pump is then used to keep this concentration constant until the tank of the reactor is completely filled, at which point the pump is then closed.

In this note, we have performed simulations for several cases in which our theoretical characterization of singular arcs is fulfilled. We have observed that it is not easy to determine which of the studied two strategies is optimal, even in cases when one could suppose that filling the reactor as quickly as possible is the best feeding strategy (for instance, in the case of Figure 1). Indeed, in many cases, this decision also depends on the initial conditions of the bioreactor. Thus, these numerical experiments can guide practitioners in choosing strategies for particular SBR settings.

It is worth noting that explicit optimal strategies cannot be directly obtained from our approach in the multi-species case. This result is a consequence of the lack of information about the number of commutations that can be employed between the above-mentioned phases (making dilutions, maintaining a constant substrate concentration and closing the pump) in an optimal strategy. This limit is the main line of research that we expect to address in future works.

Acknowledgements The authors thank Francis Mairet for fruitful discussions on the topic of this paper. We also thank two anonymous reviewers who have substantially contributed to the improved quality of this revision.

\section{References}

1. J. ANDREws: A Mathematical model for the continuous culture of microorganism utilizing inhibitory substrate, Biotechnol. \& Bioeng., 10, (1968), 707-723.

2. T. Bayen, P. Gajardo and F. Mairet: Optimal Synthesis for the Minimum Time Control Problems of Fed-Batch Bioprocesses for Growth Functions with Two Maxima, Journal of Optimization Theory and Applications, (2012), 1-33.

3. J. F. Bonnans, P. Rouchon: Commande et optimisation de systémes dynamiques, Éditions de l'École polytechnique, (2005). 
4. B. BonnARD AND I. KupKa: Théorie des singularités de lápplication entrée/sortie et optimalité des trajectoires singuliéres dans le problemé du temps minimal, Forum Mathematicum, 5, (1993), 111-159.

5. F. Ciappelloni, D. Mazouni, J. Harmand, L. Lardon : On-line supervision and control of an aerobic SBR process, Water Science and Technology, 53, (1), (2006), 169-177.

6. G. D'Ans, P. Koкотоvic And D. Gottlieb: Time optimal control for a model of bacterial growth, J. of Optimiz. Theory Appl., 7, (1971), 61-69.

7. A. Donoso-Bravo, G. Ruiz-Filippi And R. Chamy: Anaerobic treatment of low-strength wastewater with a high fraction of particulate matter in an unconventional two-phase ASBRs system, Biochemical Engineering Journal, 43, (2009), 297-302.

8. T. F. EDGAR AND L. LAPIDUS: The computation of optimal singular bang-bang control II. Nonlinear systems, AIChE Journal, American Institute of Chemical Engineers, 18, (4), (1972), 780-785.

9. L.J. Forney, W.-T. Liu, J.B. Guckert, Y. Kumagai, E. Namkung, T. Nishihara And R.J. Larson: Structure of Microbial Communities in Activated Sludge: Potential Implications for Assessing the Biodegradability of Chemicals, Ecotoxicology and Environmental Safety, 49, (1), (2001), 40-53.

10. P. Gajardo, H. Ramírez C., A. Rapaport: Minimal time sequential Batch Reactors with Bounded and Impulsive Controls for one or more species, SIAM J. on Control and Optimization, 47, (6), (2008), 2827-2856.

11. P. Gajardo, H. Ramírez C., J. C. Rodríguez: On optimal strategies for feeding in minimal time a SBR with several species, Proceeding of the 20th Mediterranean Conference on Control and Automation, D.O.I. 10.1109/MED.2012.6265667, (2012), 378-385.

12. B. S. GoH.: Necessary conditions for singular extremals involving multiple control variables, SIAM J. on Control and Optimization, 4, (4), (1966), 716-731.

13. J. Hong: Optimal substrate feeding policy for fed batch fermentation with substrate and product inhibition kinetics, Biotechnol. Bioengng., 28, (1986), 1421-1431.

14. R. L. Irvine And L. H. Ketchum: Sequencing batch reactors for biological wastewater treatment, Critical Reviews in Environmental Control, 18, (4), (1989), 255-294.

15. H. J. Kelley, R.E. Kopp And H.G. Moyer: Singular extremals, Topics in Optim., G. Leitmann ed., Academic Press, New York, (1967), 63-101.

16. A. J. KRENER : The high order maximum principle and its application to singular extremals, SIAM J. Control and Optimization, 15, 2, (1977), 256-293.

17. J. Monod : Recherches sur la croissance des cultures bactriennes, Paris, France: Hermes, (1942).

18. J. MoReno: Optimal time control of bioreactors for the wastewater treatment, Optimal Control Applications and Methods, 20, (3), (1999), 145-164.

19. L.S. Pontryagin, V.G. Boltyanskit, R.V. Gamkrelidze, and E.F. Mishchenko: The mathematical theory of optimal processes, Translated from the Russian by K. N. Trirogoff; edited by L. W. Neustadt. Interscience Publishers John Wiley \& Sons, Inc. New YorkLondon, (1962).

20. A. RAPAPORT And D. Dochain: Minimal time control of fed-batch processes with growth functions having several maxima, IEEE Trans. Automat. Control, 56, (11), (2011), 26712676.

21. C.D. Siebenthal, And R. Aris: The Application of Pontryagins Methods to the Control of a Stirred Reactor, Chem. Engng. Sci., 19, (1964), 729-746.

22. H.L. Smith and P. Waltman: The Theory of the Chemostat, Cambridge University Press, (1995). 


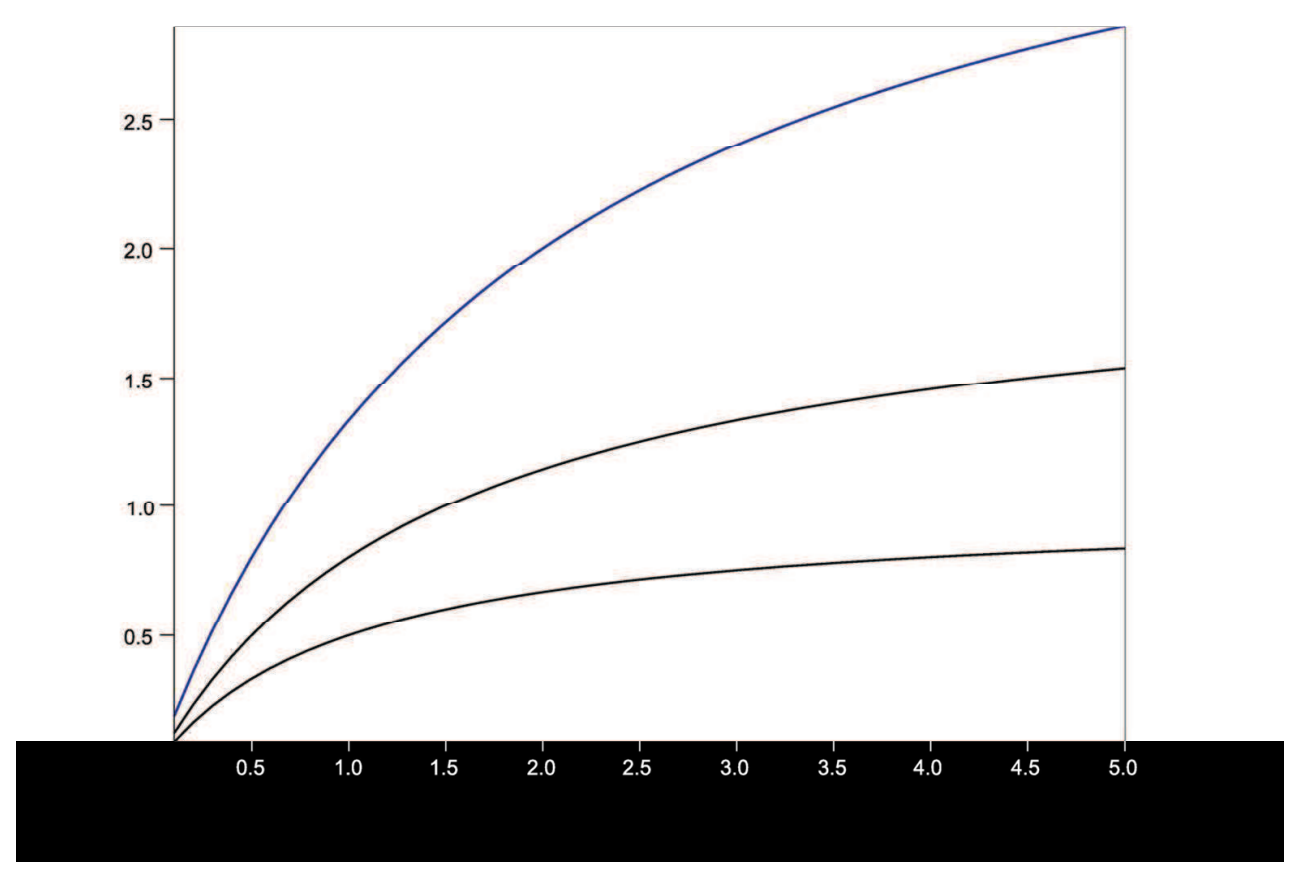

$121 \times 91 \mathrm{~mm}(300 \times 300$ DPI $)$ 
Bioprocess and Biosystems Engineering

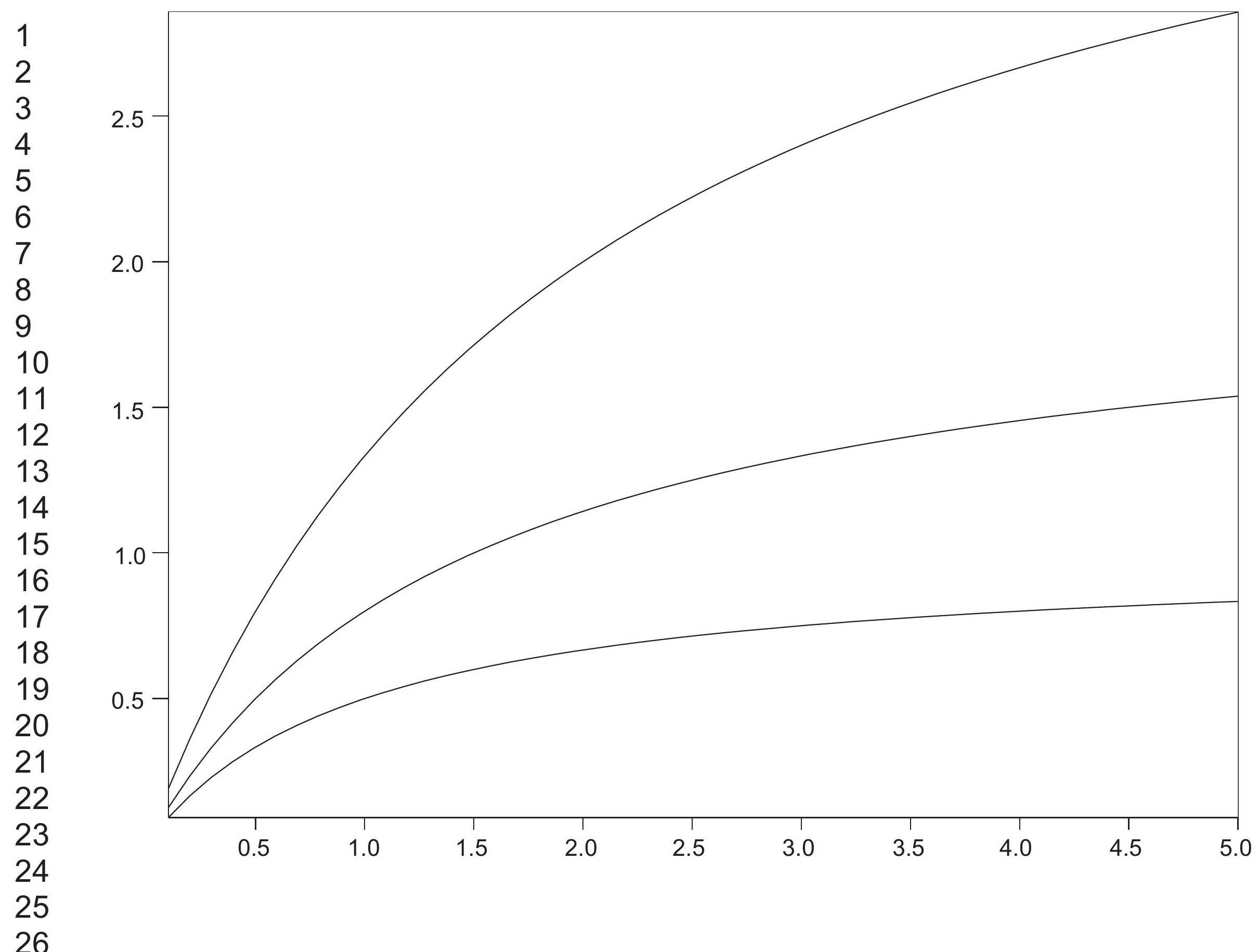

